\title{
A simplified transposon mutagenesis method to perform phenotypic forward genetic screens in cultured cells
}

\author{
Charlotte R. Feddersen ${ }^{1}$, Lexy S. Wadsworth', Eliot Y. Zhu', Hayley R. Vaughn', Andrew P. Voigt', \\ Jesse D. Riordan ${ }^{1}$ and Adam J. Dupuy ${ }^{1,2,3^{*}}$ id
}

\begin{abstract}
Background: The introduction of genome-wide shRNA and CRISPR libraries has facilitated cell-based screens to identify loss-of-function mutations associated with a phenotype of interest. Approaches to perform analogous gainof-function screens are less common, although some reports have utilized arrayed viral expression libraries or the CRISPR activation system. However, a variety of technical and logistical challenges make these approaches difficult for many labs to execute. In addition, genome-wide shRNA or CRISPR libraries typically contain of hundreds of thousands of individual engineered elements, and the associated complexity creates issues with replication and reproducibility for these methods.
\end{abstract}

Results: Here we describe a simple, reproducible approach using the SB transposon system to perform phenotypic cell-based genetic screens. This approach employs only three plasmids to perform unbiased, whole-genome transposon mutagenesis. We also describe a ligation-mediated PCR method that can be used in conjunction with the included software tools to map raw sequence data, identify candidate genes associated with phenotypes of interest, and predict the impact of recurrent transposon insertions on candidate gene function. Finally, we demonstrate the high reproducibility of our approach by having three individuals perform independent replicates of a mutagenesis screen to identify drivers of vemurafenib resistance in cultured melanoma cells.

Conclusions: Collectively, our work establishes a facile, adaptable method that can be performed by labs of any size to perform robust, genome-wide screens to identify genes that influence phenotypes of interest.

\section{Introduction}

Forward genetic screens, in which a phenotype of interest is selected from a population of mutagenized individuals, have long been viewed as a powerful tool to uncover novel components of biological systems. A variety of approaches have been used in model organisms such as yeast [12], Caenorhabditis elegans [17], and fruit flies [44]. However, forward genetic screens have been more challenging to perform in mammalian organisms, in part due to the size and complexity of mammalian genomes. Chemical mutagenesis screens have been generally useful for obtaining

\footnotetext{
* Correspondence: adam-dupuy@uiowa.edu

${ }^{1}$ Department of Anatomy \& Cell Biology, Carver College of Medicine, University of lowa, lowa City, IA 52246, USA

${ }^{2}$ Holden Comprehensive Cancer Center, University of lowa, lowa City, IA 52246, USA

Full list of author information is available at the end of the article
}

interesting mutant phenotypes in mice, but the identification of the causative genetic alterations is laborious, even with the advent of genome sequencing.

The development of genome-wide shRNA and CRISPR libraries has facilitated cell-based screens to identify loss-of-function mutations associated with specific phenotypes. Hundreds of studies have been reported using either RNAi or CRISPR screens to identify genes associated with a wide variety of phenotypes [38, 42], including extensive work to understand the vulnerabilities of cancer cell lines [28]. Fewer options exist to perform gain-of-function (e.g. over-expression) genome-wide screens in cell-based assays. The typical approach utilizes arrayed lentiviral libraries consisting of hundreds to many thousands constructs, each expressing a single open-reading frame (ORF). 
Concern remains regarding the consistency of such methods, given the substantial complexity involved with employing genome-wide libraries. Many screening libraries contain over 100,000 individual lentiviral constructs, which are typically synthesized and cloned into expression vectors in a pooled format. Inherent differences in the efficiency of vector propagation and packaging during these steps creates pools that lack homogeneity in terms of the quantity of each individual reagent. Production of arrayed libraries also requires substantial quality controls and automated liquid handling automation capabilities that most research facilities lack. Because of these issues, such genome-wide screens must be carefully designed and executed, including the use of complex statistical models to interpret and remove the substantial number of false positive hits. Ultimately, the complexity and expense associated with existing genome-wide screening approaches limits the ability of independent research groups to conduct novel screens or replicate previously-reported results.

Compared to complex genome-wide screening methods that individually target elements at the genome scale, insertional mutagenesis screens are generally much simpler. Retroviral insertional mutagenesis has been used to select for phenotypes and mutations of interest in cultured cells $[14,19,22]$. However, retroviral vectors typically exhibit significant insertion bias, and proviral integration can have complex effects on gene expression, thus limiting the utility of viral insertional mutagenesis. By contrast, transposon systems, such as SB and piggyBac, have become more commonly used for insertional mutagenesis due to their flexible design and reduced integration site bias. While transposon mutagenesis has been used to perform phenotypic selection in cells ex vivo $[6,15,23]$, it has more frequently been employed in engineered mouse models of cancer [35], likely due to the relative inefficiency of mutagenesis when both transposon and transposase vectors must be introduced independently to cells in culture.

Here we describe a novel method to perform simple, phenotype-driven genome-wide genetic screens in cultured cells using a hyperactive version of the SB transposon system. Unlike other genome-wide screening methods [16, 24, 43, 49], ours consists of only three plasmids that are used to carry out mutagenesis in cultured cells. Following phenotypic selection of mutagenized cells, a simple ligation-mediated PCR method (Additional file 2: Supplemental Methods) prepares libraries that can be multiplexed and directly sequenced on the Illumina platform. We developed a series of software tools to take raw sequence data as input and perform trimming, mapping, and filtering of transposon integration sites. We also report an updated gene-centric (biased for analysis of annotated genes) common insertion site (gCIS) analysis tool modified from a previously described method to analyze transposon integration data from SB models of cancer [5] that predicts the functional impact of transposon insertions on the adjacent genes. Collectively, our methods and analytical tools provide labs of virtually any size with the ability to independently perform genome-wide phenotypic screens in cultured cells.

\section{Results}

Transposon mutagenesis in cultured cells

We considered several factors when setting out to develop a cell-based forward mutagenesis screening approach. Ideally, a plasmid-based system provides the greatest flexibility to mutagenize any cell line that can be transfected with moderate to high efficiency. While our initial efforts with the SB11 transposase enzyme [13] did not provide sufficient mutagenesis efficiency for screens (not shown), a hyperactive version of the SB transposase with $\sim 100$ fold increased activity, SB100X [32], was sufficient for screening purposes. We generated a piggyBac vector to create a simple, viral-free method to establish stable expression of the SB transposase in mammalian cells by simply co-transfecting the SB100X vector with a second vector encoding a hyperactive version of the piggyBac transposase (hyPBase) [54](Fig. 1). Once cells stably expressing SB100X are generated, transposon mutagenesis is performed by simply transfecting a mutagenic transposon (e.g. pT2-Onc, pT2-Onc2, pT2-Onc3) [7, 9, 10]. The SB100X enzyme then mobilizes mutagenic transposons from the transfected plasmid into the cellular genome. Once integrated, the transposons can impact gene expression through a few known mechanisms [8].

To characterize our forward mutagenesis screening system, we performed a screen to identify novel drivers of resistance to the BRAF inhibitor vemurafenib in cultured melanoma cells. We selected the A375 melanoma cell based on its recurrent usage in shRNA/sgRNA screens to identify drivers of vemurafenib resistance [18, 43, 50]. A population of A375 cells stably expressing SB100X transposase (A375-SB100X) was produced and subsequently transfected with either the mutagenic pT2Onc3 transposon vector [10] or a control EGFP expression plasmid. Cells were grown for $48 \mathrm{~h}$ to facilitate integration of mutagenic transposons, independent plates of mutagenized or control cells were pooled, and $1 \times 10^{5}$ cells were seeded onto 6-well plates. Twelve to twentyfour hours post-plating, cells were placed under drug selection in 3,5 , or $10 \mu \mathrm{M}$ vemurafenib or vehicle control (dimethylsulfoxide). Media was changed twice weekly, and wells were monitored for the emergence of resistant colonies. Both mutagenized and control plates were harvested and stained when resistant colonies were plainly observable in control wells. 
1) create stable SB100X-expressing cells

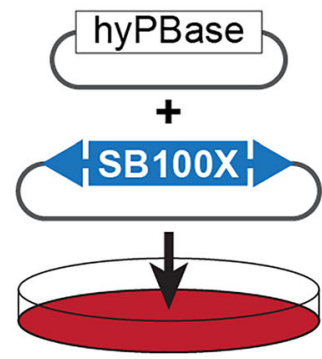

2) transfect SB100X-expressing cells with mutagenic transposon, allow cells to recover, and apply phenotype selection

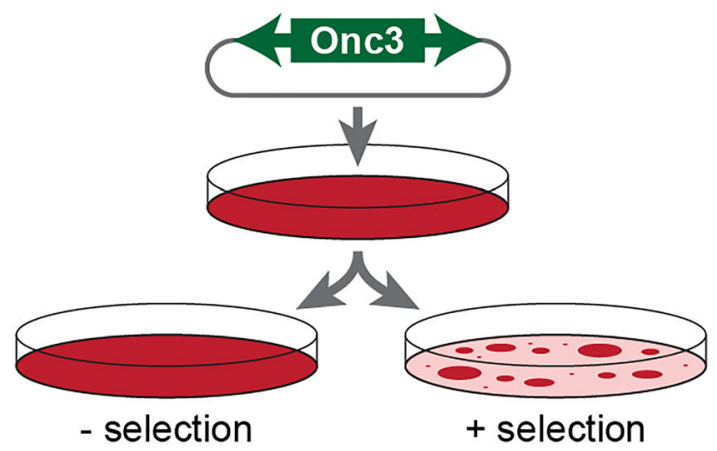

3) collect mutagenized cells, isolate genomic DNA, create libraries using ligation-mediated PCR, multiplex libraries and sequence

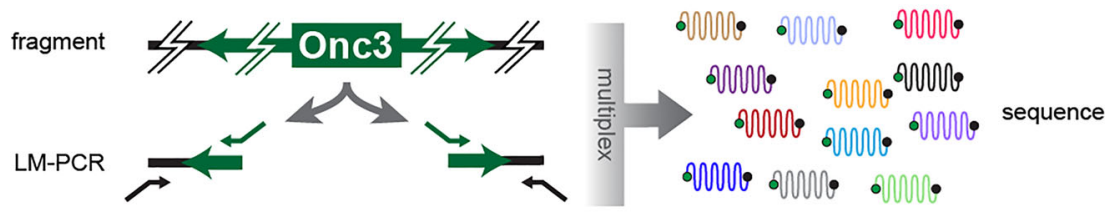

4) trim adaptors, map to reference genome, and filter sequences to remove low abundance reads
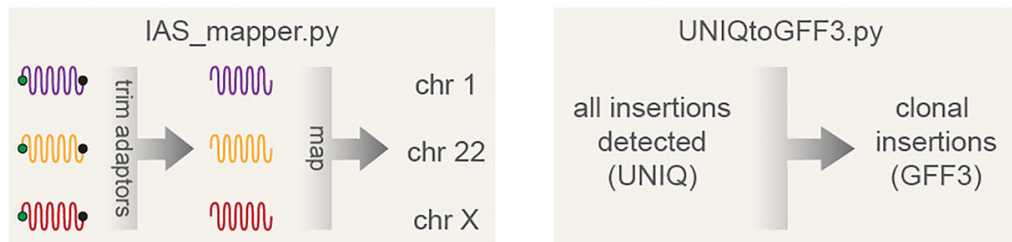

5) combine results from each treatment group and perform genecentric common insertion site analysis

\begin{tabular}{|c|c|c|c|c|}
\hline \multicolumn{5}{|c|}{ gCIS_v2.py } \\
\hline combined GFF3 & gene & $p$-value & FDR & mechanism \\
\hline $\begin{array}{l}\text { sample } 1 \text { sample } 4 \\
\text { sample } 2 \text { sample } 5 \\
\text { sample } 3 \text { sample } 6\end{array}$ & $\begin{array}{l}\text { gene A } \\
\text { gene B } \\
\text { gene C }\end{array}$ & $\begin{array}{l}x x x \\
y y y \\
z z z\end{array}$ & $\begin{array}{l}x x x \\
y y y \\
z z z\end{array}$ & $\begin{array}{l}\text { over-expression } \\
\text { over-expression } \\
\text { disruption }\end{array}$ \\
\hline
\end{tabular}

Fig. 1 Overview of cell-based Sleeping Beauty (SB) mutagenesis screens. (1) Cells are engineered to express the SB100X transposase. This is done using a piggyBac transposon expression system using the hyPBase transposase to stably deliver the SB100X transgene. (2) Once SB100Xexpressing cells are selected, cells are transfected with the T2-Onc3 plasmid, or similar mutagenic SB transposon vector, and cells with the desired phenotype are selected from among the population of mutagenized cells. (3) Genomic DNA is collected from each population of selected and control cells, and each DNA sample is subjected to a ligation-mediated PCR approach to isolate the transposon-genome junctions. Individual LMPCR libraries are then multiplexed and sequenced on the Illumina platform. (4) Raw sequences are trimmed, mapped to the reference genome, and filtered based on user-defined criteria. (5) Finally, filtered sequences are combined and analyzed to identify candidate genes enriched for transposon insertions in selected but not control cells 
Pilot experiments indicated that $5 \mu \mathrm{M}$ vemurafenib was the optimal drug concentration to select for resistant SBmutagenized A375 cells, based on several observations. At $3 \mu \mathrm{M}$ vemurafenib, resistant colonies emerged at roughly the same time in treated and control wells, indicating an insufficient stringency to distinguish between spontaneous resistance and resistance driven by transposon mutagenesis. While $10 \mu \mathrm{M}$ vemurafenib greatly reduced the frequency of spontaneous resistance in the control wells, colonies that eventually emerged in mutagenized cells were fewer and smaller than those that grew at lower drug concentrations. In contrast, at $5 \mu \mathrm{M}$ vemurafenib, mutagenized A375 cells produced large colonies in only 10-14 days, while control cells did not produce large resistant clones in the same timeframe (Fig. 2). Based on these results, we selected a $5 \mu \mathrm{M}$ vemurafenib dose to perform our mutagenesis screen for drivers of vemurafenib resistance.

A375-SB100X cells were transfected with the pT2Onc3 transposon plasmid to initialize mutagenesis. Twenty-four hours post-transfection, $10 \mathrm{~cm}$ plates were seeded with $1 \times 10^{6}$ cells and allowed to recover for $12-$ $24 \mathrm{~h}$. After recovery, each plate was treated with either $5 \mu \mathrm{M}$ vemurafenib or dimethylsulfoxide (i.e. vehicle control). While vehicle-treated cells expanded rapidly and reached confluency $\sim 3$ days after plating, drug-resistant colonies emerged within 10-14 days after the addition of drug, as previously observed in the pilot study. Importantly, this experiment was independently conducted by three different members of the lab with similar results (see Methods). In all cases, vemurafenib resistant colonies emerged in mutagenized cells in 10-14 days while control cells did not develop spontaneous resistance in the same timeframe.

\section{Identification of transposon insertion sites}

Next, we sought to determine if recurrent transposoninduced mutations could be identified in resistant colonies. As picking individual colonies would significantly limit throughput, we instead harvested independent plates of cells as pooled populations, as is typically done with genome-wide shRNA and CRISPR screens. We collected pooled cell populations from each independent $10 \mathrm{~cm}$ plate and extracted genomic DNA. Fragments containing the transposon/genome junctions were amplified via ligation-mediated PCR (LM-PCR) and sequenced using the Illumina HiSeq 4000 platform (Additional file 2: Supplemental Methods). A single FASTQ file was generated for each population of vemurafenib- $(n=69)$ and vehicletreated $(n=15)$ cell population. We generated an informatics pipeline (IAS_mapper) to processes each FASTQ file, trimming the residual transposon and adaptor sequences and mapping trimmed reads to the human reference genome (GRCh38). Although we designed the IAS_mapper pipeline to utilize adaptor trimming (cutadapt) [31] and mapping (HISAT2)[21] methods that can be run on a standard workstation, the software can be modified to run on a parallel processing environment to decrease processing time.

\section{SB100X-mediated transposition results in numerous integration events per cell}

We sought to determine the efficiency of mutagenesis at the cellular level to get an estimate of the number of insertion events that can be achieved with our approach. To do this, we isolated and expanded eight individual vemurafenib-resistant colonies derived from three independent plates. Following expansion, we verified the drug-resistance of a subset of colonies in a 10-day growth assay (Additional file 1: Figure S1). We then identified transposon integration sites as described above. Interestingly, the number of insertions within each colony ranged from 10 to 135 (Fig. 3). Colonies harboring large numbers of insertions are likely to be mixed populations due to collection and isolation process. However, it is also possible that single cells harbor $>100$ transposon insertion events, similar to SB-induced leukemias we have previously described [3]. Regardless, these results indicate that transposon mobilization from transfected plasmids is highly efficient, resulting in numerous insertional mutation events per cell.

\section{Gene-centric common insertion site analysis identifies recurrently mutated genes in selected cells}

The identification of candidate genes in SB screens essentially relies on the identification of genes that suffer transposon insertion events at a rate that exceeds the expected background rate of mutation, based on the experimental parameters. While this concept is straightforward, most integrating vector systems display insertion site bias, often favoring regions near transcription start sites. Therefore, integration site bias can produce a high rate of false-positive gene candidates. A variety of prior studies have shown that the SB transposase exhibits little integration site bias relative to other integrating vectors, requiring only a TA dinucleotide for insertion [1, 27, 39, 41, 48, 51, 53]. This feature of the SB system is beneficial because it 1) allows SB mutagenesis to access virtually the entire genome and 2) simplifies the analysis of SB integration data by eliminating the need to produce complex statistical models that account for bias.

We have previously described a method to identify genes that harbor recurrent $\mathrm{SB}$ transposon insertion events in association with a phenotype of interest [5]. This method, referred to as gene-centric common insertion site (gCIS) analysis, performs a simple chi-squared test to compare the number of observed insertion events in a given gene to the number of expected insertion events based the number of TA sites within the gene and the total number of insertion events identified in the data set. The initial gCIS method was designed to analyze insertion data derived from SB-induced tumors 


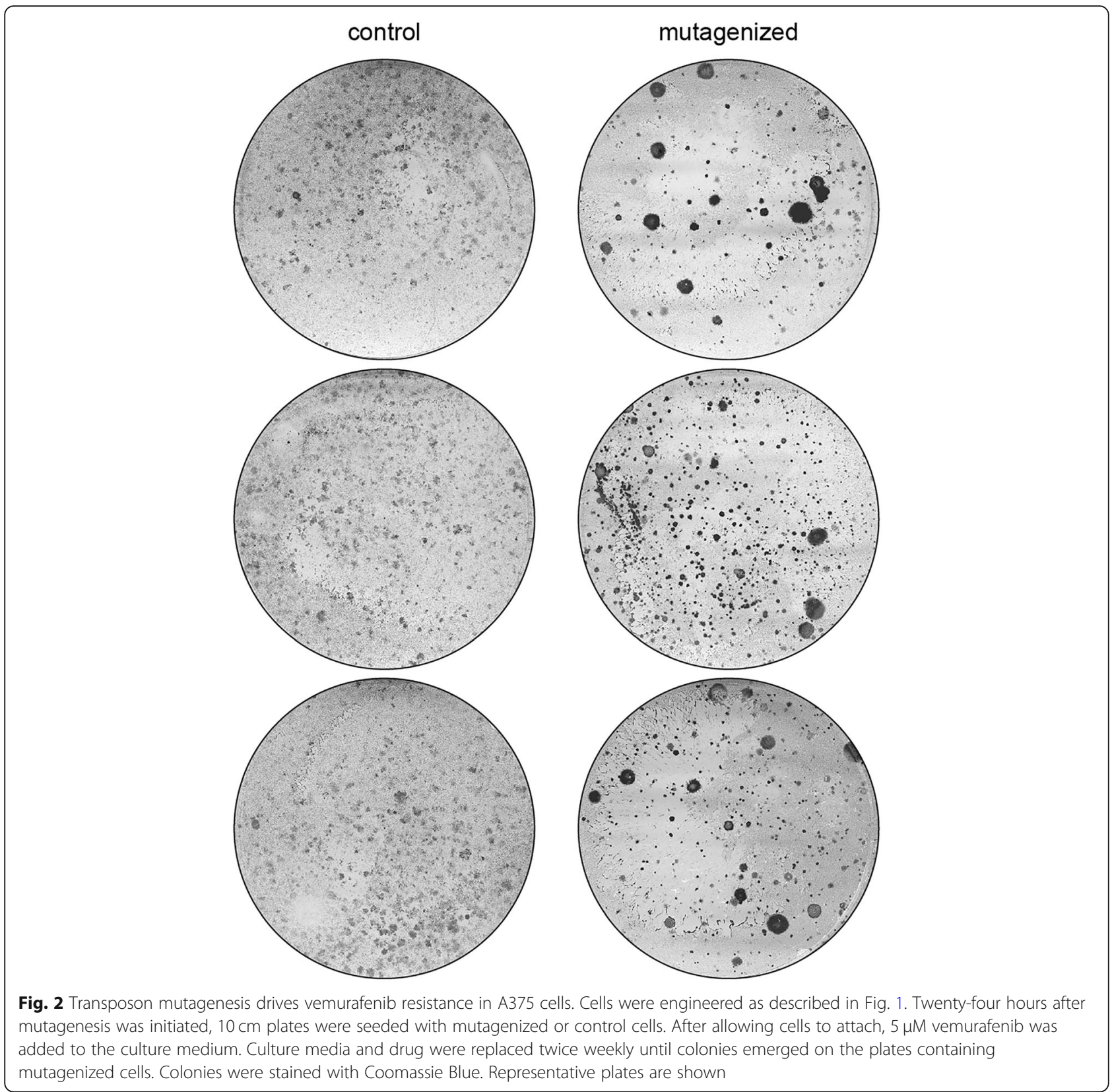

in engineered mouse models. As a consequence, the pipeline assumes each analyzed sample is a single, clonally-derived cell population (i.e. a tumor), not a pooled population of unrelated clones.

We developed a new gCIS method (gCIS2) to analyze transposon insertion data from cell-based phenotypic screens, such as our vemurafenib resistance screen performed in A375 melanoma cells (for detailed description see Additional file 2: Supplemental Methods). Given that we used a pooled sample preparation to facilitate higher throughput for the screen, we were unable to determine which insertion sites co-occur within the same cell clone. Therefore, we evaluated each unique insertion site as an independent event. This approach ignores potential interactions between insertions that co-occur in the same cell and instead assumes that single insertion events can provide the desired phenotype, allowing evaluation of cell pools containing an unknown number of clonal populations.

We next analyzed insertion site data from the vemurafeniband vehicle-treated A375 cell populations using the gCIS2 tool (Additional file 3: Table S1). The pipeline identified 11,094 unique transposon insertion events in 7598 RefSeq genes, including the transcription unit and promoter region - defined as 40 kilobases upstream of the start site. Overall, nine genes were significantly enriched for transposon insertions (false 

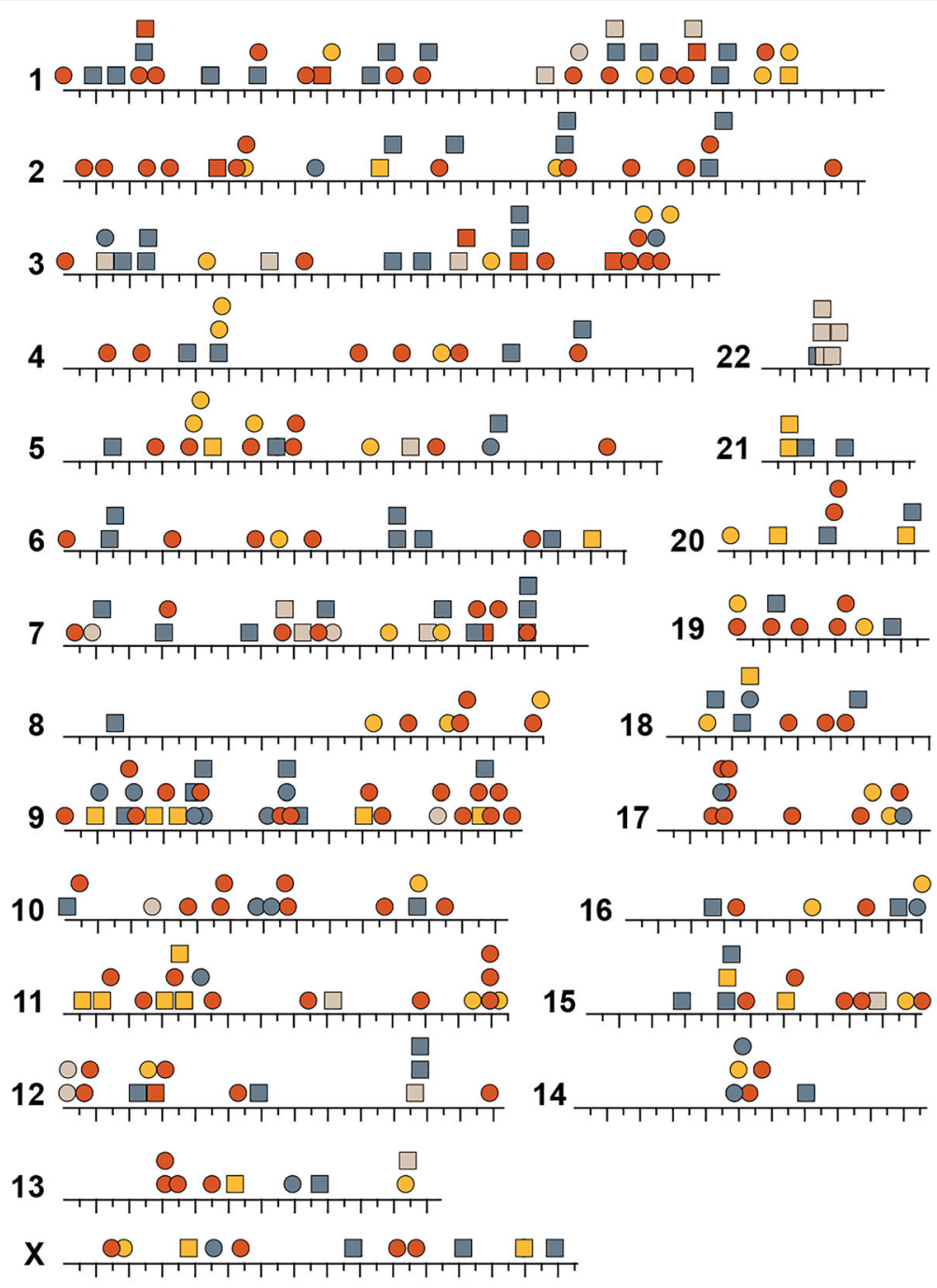

\begin{tabular}{|c|c|c|c|}
\hline clone & \# of insertions & clone & \# of insertions \\
\hline A2 & 23 & A6 & 40 \\
\hline$\square$ A3 & 82 & $\square$ B3 & 27 \\
\hline A4 & 135 & B5 & 10 \\
\hline$\square$ A5 & 10 & $\square \mathrm{C} 5$ & 20 \\
\hline
\end{tabular}

Fig. 3 Comparison of transposon insertion sites in a series of vemurafenib-resistant A375 colonies. Eight distinct vemurafenib-resistant colonies were isolated from three independent populations of SB-mutagenized A375 cells. Each colony was expanded and transposon insertion sites were identified (see Fig. 1). Between 10 and 135 insertion events were present in each expanded colony. In general, transposon insertion sites were distributed across the genome, suggesting that transposon mutagenesis is capable of performing genome-wide screens

discovery rate $\leq 1 \times 10^{-5}$ ), were unique to the vemurafenibtreated population (see Methods) and showed a transposon distribution pattern and orientation trend consistent with either over-expression or disruption. For example, the pipeline identified 162 independent transposon insertions within the 5' region of the VAV1 locus. Nearly all of the transposons (161/ 162) inserted in the promoter region or first intron of the locus, and the vast majority (88\%) of transposons inserted in the same transcriptional orientation as the VAV1 gene, suggesting that over-expression of VAV1 is associated with vemurafenib resistance in A375 cells.

\section{Functional prediction of transposon effects on candidate genes}

Prior insertional mutagenesis screen analysis pipelines, including our original gCIS method, identify candidate 
genes by searching for clusters of transposon insertion events within the genome but do not attempt to predict the impact of transposon insertion on the affected genes. Mutagenic SB transposons were developed to modify expression of endogenous genes in specific ways depending on their relative position and orientation, as previously described [8]. This feature, combined with our experience analyzing insertion site data from $15 \mathrm{SB}$-induced models of cancer $[2,3,20,29,30,33,34,37,40,41,45-47,52,55]$, led us to develop an algorithm to predict the functional impact that recurrent transposon insertion has on a given gene. This approach evaluates clustered transposon insertions within each candidate locus to determine if there is a bias for insertion in the same orientation as the gene, indicative of an over-expression mechanism, or if the transposons are randomly orientated, suggesting a gene disruption mechanism (see Additional file 2: Supplemental Methods). Of the nine genes identified by gCIS2, eight were predicted to drive resistance through over-expression and one was predicted to drive resistance via gene disruption (Additional file 3: Table S1). Importantly, we have recently described experiments that functionally validated the role of four of these candidates (VAV1, MCF2, BRAF, RAF1) in driving vemurafenib resistance in human melanoma cell lines [11].

We next evaluated the eight genes predicted to drive vemurafenib resistance via over-expression, as determined by gCIS2. Transposon orientation bias was evident in each of these genes, and in the majority of cases the cluster of transposon insertions was centered in the $5^{\prime}$ region. For two genes, $B R A F$ and $R A F 1$, transposons were clustered within the gene body well downstream of the promoter, a pattern predicted to drive over-expression of an mRNA encoding an $\mathrm{N}$-terminally truncated protein (Additional file 1: Figure S2). We modified the gCIS2 method to facilitate the identification of rare genes in which a similar protein truncation mechanism may be occurring, evaluating the distribution of transposon insertion sites across each gene to calculate the skewness and kurtosis (Fig. 4a). Skewness provides an indication of clustering near the $5^{\prime}$ (positive skewness) or 3' (negative skewness) region of the gene. Kurtosis provides an indication of how tight (positive kurtosis) or loose (negative kurtosis) the distribution of transposon insertions is within the gene (Fig. 4a).

\section{Evaluation of gCIS2 performance using simulated data sets}

We have previously compared SB transposon integration data to matched sets of randomly selected sets of TA dinucleotide sites to detect non-random patterns in the experimental data set [39]. Using this approach, we generated twenty independent, simulated data sets by replacing each transposon insertion event with a randomly selected TA dinucleotide site from the human reference genome (GRCh38). Each simulated data set was then analyzed by gCIS2.

As described above, gCIS2 identified nine candidate resistance genes in the experimental data set that met the criteria defined by the pipeline (i.e. FDR $\leq 1 \times 10^{-5}$ with conserved function prediction). By contrast, gCIS2 did not identify genes that met the same criteria in the twenty simulated random data sets. This result is not surprising given that the gCIS2 method assumes a random distribution of transposon insertion sites.
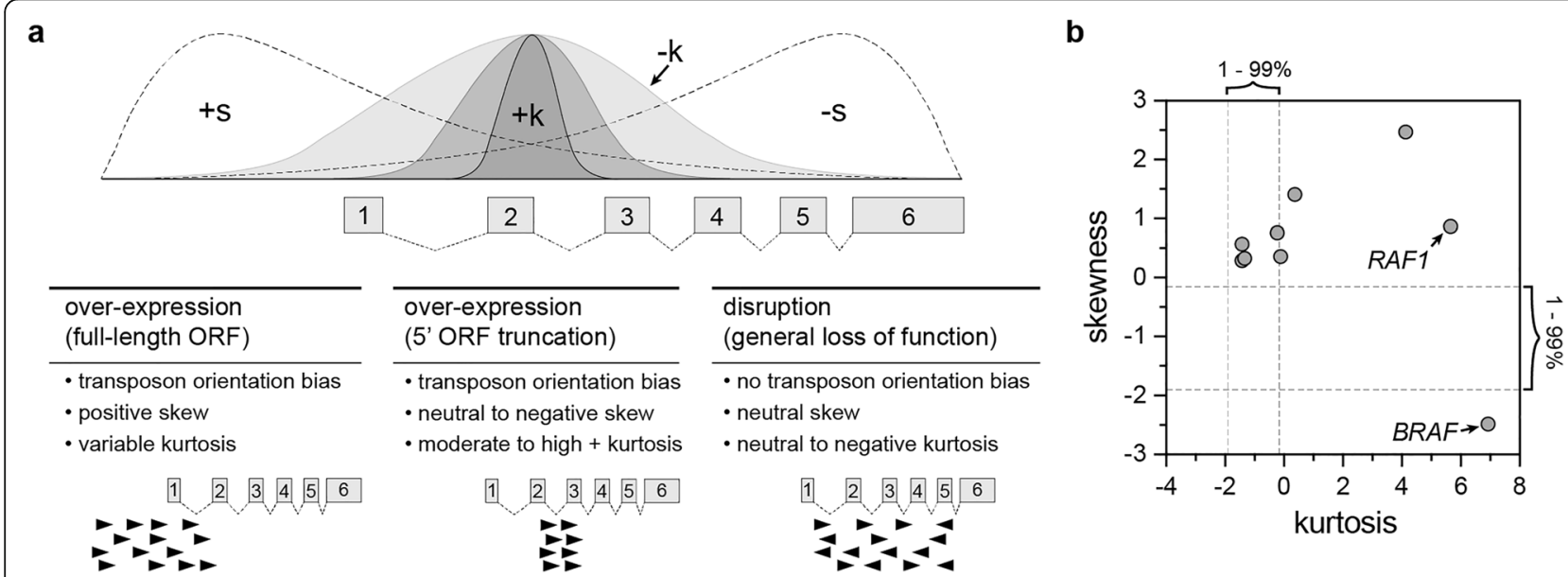

Fig. 4 Predicting the functional impact of transposon insertion on individual candidate genes. The gCIS2 pipeline employs an algorithm for each gene that has $\geq 5$ independent insertions to predict the impact of transposon insertion on the gene (i.e. over-expression or gene disruption). a The gCIS2 pipeline also determines the skewness and kurtosis values for the distribution of transposon insertions across the gene. These values can be evaluated by the user to further distinguish between different mechanisms of over-expression (i.e. full-length vs. 5'truncation). $\mathbf{b}$ Actual skewness and kurtosis values are shown for the candidate drivers of vemurafenib resistance identified in A375 cells. [dashed lines indicate the 199\% intervals for skewness and kurtosis obtained from the analysis of 20 independent simulated data sets (see Methods)] 
Next, we compared skewness and kurtosis values between the experimental and simulated data sets. We determined the mean skewness and kurtosis values for the 1 st and 99th percentiles among the twenty simulated data sets (Additional file 1: Figure S3). Generally, the experimental data set had limits similar to the simulated data sets for both skewness and kurtosis but had significantly greater values, particularly for kurtosis. This result demonstrates that the experimental data set contains more tightly clustered transposons within some genes, indicative of strong selective pressure for integration within a relatively small genomic region within each locus.

The gCIS2 pipeline calculates both skewness and kurtosis for each gene analyzed, but these values are not used to make predictions about the functional impact of transposon insertion on a given gene. Instead, they can be used to evaluate the extent to which transposons show a conserved functional mechanism such as over-expression of a full length open-reading frame (ORF), over-expression of a truncated ORF, or gene disruption (Fig. 4a). For example, the skewness and kurtosis values calculated for each of the nine candidate genes identified by gCIS2 in the experimental data set shows particularly high kurtosis values for both RAF1 and BRAF (Fig. 4b). This feature could be useful in identifying cases in which transposoninduced gene truncation is associated with the selected phenotype, vemurafenib resistance in this case.

\section{Independent replicates of vemurafenib-resistance screen yield nearly identical results}

A major criticism of genome-wide screens using shRNA and CRISPR is the lack of reproducibility between independent experiments. For example, three independent genome-wide loss-of-function screens to identify drivers of vemurafenib resistance in A375 cells have been reported [26, 43, 50]. Of the 34 candidate genes reported in these publications, only NF1 was identified by all three. As previously mentioned, three different individuals in this study performed our vemurafenib resistance SB mutagenesis screen in A375 cells using the same procedure. In part, this strategy was used to determine the reproducibility of our transposon screening method. Analysis of the entire data set identified nine candidate genes (Additional file 3: Table S1). Encouragingly, 6 of these 9 genes were identified as significant in all three replicates of the screen, despite significant variations in the sample size (Fig. 5). The remaining three genes were identified as significant in two of three screens. This finding provides evidence that the transposon mutagenesis method described here provides a high degree of reproducibility.

\section{Discussion}

Here we describe the development of both the molecular and bioinformatic tools needed to perform SB transposonbased phenotypic screens in cultured mammalian cells.
Previously, the application of SB transposon mutagenesis was mostly restricted to genetically engineered mouse models of cancer. Our prior attempts at performing cellbased mutagenesis using earlier versions of the SB transposase failed to produce vemurafenib-resistant A375 cells at a frequency above the spontaneous rate of resistance (not shown), presumably due to insufficient transposition efficiency. However, we show here that the use of the hyperactive SB100X transposase mediates multiple transposon insertion events per cell when transposons are introduced as plasmids via standard transfection (Fig. 3).

Our first application of this mutagenesis approach was to derive human melanoma cells resistant to the BRAF inhibitor vemurafenib (Fig. 2) [11]. This screen identified nine candidate genes as drivers of vemurafenib resistance

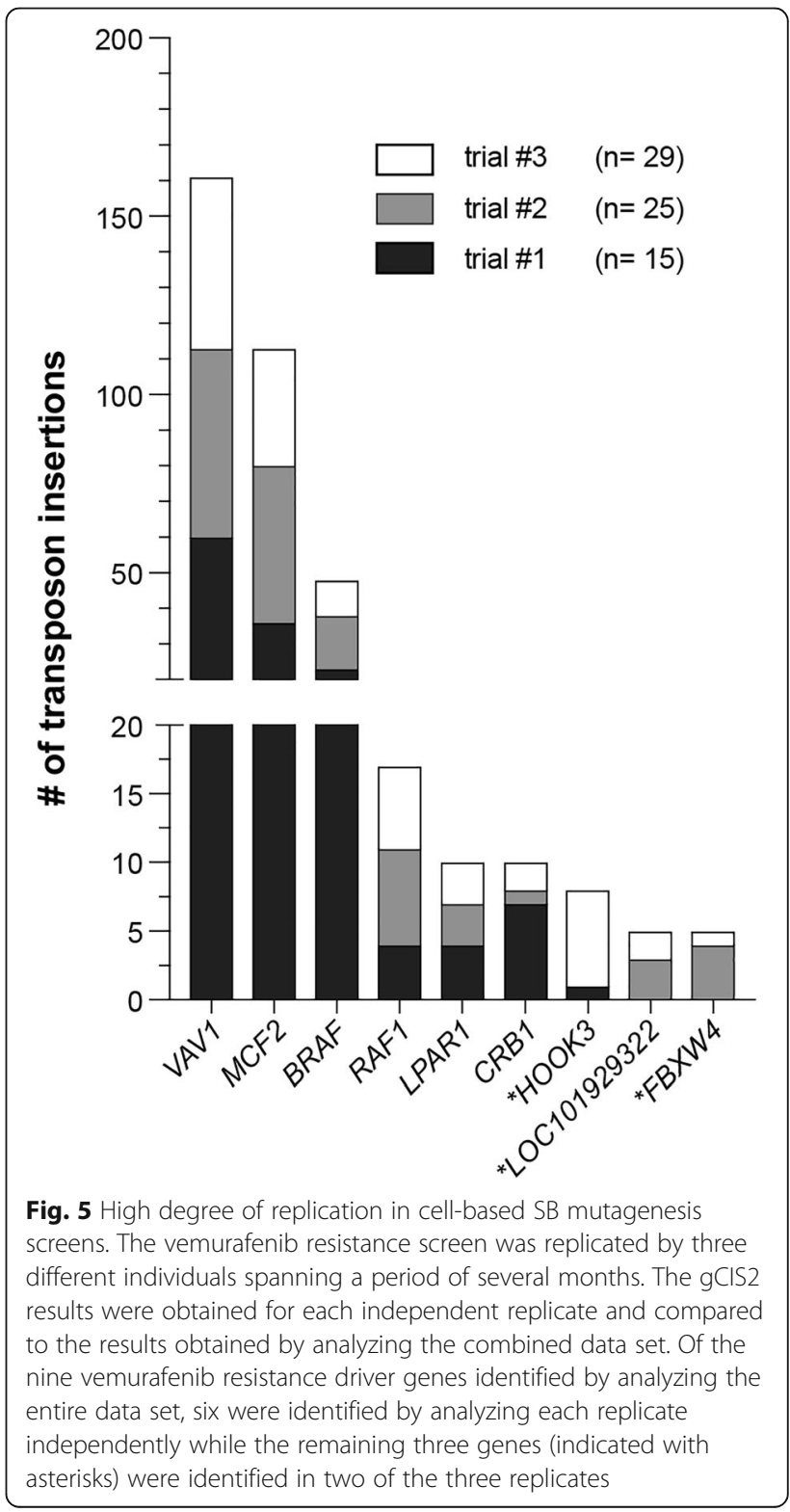


in A375 cells (Additional file 3: Table S1). A major advantage of our SB mutagenesis approach is its methodological simplicity, as demonstrated by the ability of three different individuals to perform the screen and obtain highly similar results (Fig. 5). This outcome was achieved with the methods described here in detail using commerciallyavailable reagents.

This flexible approach can be easily adapted to identify genes associated with any selectable phenotype. In general, we envision the use of our transposon-based screening method to work in the following manner. The process of generating mutagenized cells would stay relatively similar across experiments, with slight variations to achieve optimal transfection in the cell line being used. After mutagenesis is performed, the selection mechanism can be changed to fit the investigators' biological question. A drug selection, like we describe here, is a simple application. Nevertheless, other selection mechanisms could be employed like cell sorting based on one or more parameters, such as expression of a reporter gene or surface marker, cell motility or invasion, or rescue experiments to restore cell viability following a particular insult. While the potential phenotypic screens that could be performed using this approach are seemingly endless, each screen will require optimization. There are a few areas to be considered when designing a new screen.

The selection method must be optimized to reduce the background signal in non-mutagenized cells (i.e. reduce the rate of spontaneous phenotype acquisition). For example, we initially conducted an experiment to determine the optimal concentration of vemurafenib that was high enough to slow the development of spontaneous drug resistance in A375 cells while still allowing for the emergence of resistant colonies in the mutagenized cell population (Fig. 2). Choosing the appropriate selection stringency is extremely important and will vary by screen. Parameters to consider when choosing a selection dose are the rate of spontaneous phenotype acquisition and the hypothesized frequency of phenotype driver mutations. If the spontaneous rate of phenotype acquisition is high, increasing the stringency or lowering plating density can help prevent the emergence of spontaneous phenotype acquisition (i.e. non-transposon driven). Conversely, if the hypothesized frequency of mutation is low, lowering stringency may allow for weaker drivers to survive and proliferate. Regardless, the selection strategy for each screening method must be carefully considered.

Control cell populations must be included in each screen, and their production should be carefully considered. In addition to subjecting non-mutagenized controls to selection, which provides an assessment of the rate of spontaneous phenotype acquisition, a cell population that is mutagenized in the absence of selection is required. Ideally, these control cells should be derived from the same population of mutagenized cells that undergo phenotypic selection (see step 2 in Fig. 1). To the greatest extent possible, cells in the control and experimental populations should be treated identically, aside from the phenotypic selection present in the experimental population. With this design, the control cells will facilitate the identification of false-positive gene hits (i.e. mutations that are enriched in the absence of the experimental selection). These false-positive gene hits can emerge from a variety of sources such as indirect selection for mutations that drive cell proliferation and/or viability, the production of PCR artifacts during the LM-PCR step prior to sequencing, and mapping artifacts produced during the sequence analysis step. Regardless of the source, analysis of the control cell population will identify genes that are not specifically associated with the phenotype.

When designing this method, we considered the major technical and logistical limitations of current genomewide genetic screening techniques (e.g. shRNA, CRISPR, CRISPR). Most genome-wide RNAi and CRISPR methods often utilize pooled lentiviral libraries consisting of tens of thousands of individual vectors, each expressing a single shRNA or sgRNA [16, 24, 43, 49]. The propagation and viral packaging of this type of pooled library requires careful quality control measures to ensure the complexity of the library is maintained. By contrast, our cell-based SB mutagenesis approach requires only three plasmids, making it a simple and cost-effective method.

The first application of the cell-based SB mutagenesis approach was a drug resistance screen to identify drivers of vemurafenib resistance in A375 melanoma cells. This screen largely identified genes that drive resistance to vemurafenib when over-expressed [11]. In hindsight, the absence of loss-of-function candidates in our screen is not surprising since the relatively low number of independent transposon insertion events per cell and is likely insufficient to inactivate all alleles of a single gene in a cell. In this sense, our SB mutagenesis approach is more comparable to ORF screens or genome-wide screens that utilize the CRISPR activation (CRISPRa) approach [4, 25].

A recent CRISPRa screen performed by Konermann et al. identified candidate drug resistance drivers in A375 cells treated with PLX4720, a BRAF inhibitor that is similar to vemurafenib (e.g. PLX4032) [25]. Of the eight genes identified in our vemurafenib resistance screen that were also evaluated by Konermann et al., only one gene (LPAR1) was identified as a significant driver in both screens. However, two of the major drivers identified by SB mutagenesis only, BRAF and RAF1, appeared to require $\mathrm{N}$-terminal truncation as part of the resistance mechanism (Additional file 1: Figure S2) [11]. It is important to note that $\mathrm{N}$-terminal truncation of $\mathrm{BRAF}^{\mathrm{V} 600 \mathrm{E}}$ has previously been associated with 
vemurafenib resistance in human melanoma patients [36]. Thus, SB mutagenesis identified a clinicallyrelevant mechanism that was missed by the CRISPRa method. This represents a limitation of the CRISPRa method in that this approach can only interrogate annotated transcriptional start sites while SB mutagenesis can perform a more unbiased screen.

Forward genetic screens allow for the association of specific genes with phenotypes of interest, and they have been indispensable in furthering our understanding of biology. While genome-wide approaches that employ RNAi or CRISPR have facilitated more rapid, cell-based screens, the complexity of performing and analyzing these experiments is beyond the capabilities of most labs. We provide here the reagents and tools necessary for virtually any lab to perform cell-based SB screens.

\section{Methods}

\section{Mutagenesis of cultured A375 cells}

Cells were stably transfected via Effectene (Qiagen) coupled with a piggyBac transposase integration system ( $\mathrm{Li}$ et al., 2013) consisting of a piggyBac transposase-expressing plasmid and an Ef1 $\alpha$-SB100X transgene embedded within a piggyBac transposon. After puromycin selection, SB100Xpositive cells were transfected with the pT2/Onc3 transposon plasmid [10], and $1 \times 10^{6}$ mutagenized cells were plated on $10 \mathrm{~cm}$ plates $48 \mathrm{~h}$ later. Cells were subsequently treated with vemurafenib or vehicle (DMSO, $0.2 \%$ ) $24 \mathrm{~h}$ after plating. Drug/vehicle was renewed every 3-4 days. Upon reaching confluency (approximately 3 days post-plating), vehicle plates were collected via trypsinization. This process was repeated independently at three different times by Hayley Vaughn (trial \#1, $n=15$ ), Andrew Voigt (trial \#2, $n=25$ ), and Eliot Zhu (trial \#3, $n=29$ ).

For clonal isolation, a pipette tip was lightly placed in the center of a resistant colony and then dipped in media contained in a 24-well dish. Six colonies from three plates were collected in this manner for a total of 18 independent colonies. Two colonies failed to grow during the expansion phase and only eight were genetically profiled.

To identify common transposon insertion sites across plates of resistant cells or from expanded clonal populations, genomic DNA from each plate was collected via GenElute $^{\mathrm{mt}}$ Mammalian Genomic DNA Miniprep Kit (Sigma). DNA fragments containing transposon/genome junctions were amplified via ligation mediated PCR and sequenced using the Illumina Hi-Seq 4000 platform.

\section{Colony staining}

SB100X-A375 cells $\left(1 \times 10^{5}\right)$ transfected with either pEGFP or pT2/Onc3 were stained with Coomassie Brilliant Blue after ethanol fixation after 25 days of drug treatment. Colonies were counted with the GelCount $^{\text {tm }}$ Colony Counter (Oxford Optronix).

\section{Cell culture}

All cells were grown in DMEM (Gibco) supplemented with penicillin/streptomycin (Gibco) and 10\% FBS (Atlanta Biologicals).

\section{Generation of simulated data sets}

Independent data sets were generated by randomly selecting TA sites on each chromosome. The size of each random TA set was matched to the observed number of insertion events on each chromosome in the experimental data set.

\section{Access to analysis tools}

All tools, including accompanying documentation, described in this manuscript can be acquired through GitHub (https://github.com/addupuy/IAS.git).

\section{Additional files}

Additional file 1: Figures S1-S3. Supplemental figures showing additional analysis of expanded vemurafenib-resistant clones and distribution of skewness and kurtosis values among the transposon cluster sites. (PDF $225 \mathrm{~kb}$ )

Additional file 2: Additional methods. Detailed method for preparing ligation-mediated PCR libraries, overview of the IAS tools. (PDF $179 \mathrm{~kb}$ )

Additional file 3: Table S1. Results from vemurafenib resistance screen in A375 cells. (XLSX $14 \mathrm{~kb}$ )

\section{Acknowledgements}

The authors would like to thank Drs. Michael Henry and Christopher Stipp for their careful review and editing of this manuscript.

Authors' contributions

CRF, LSW, EYZ, HRV, and APV contributed experimental data for the study. AJD designed and developed the bioinformatic tools described in the manuscript. JDR evaluated and provided critical evaluation of the bioinformatic tools. CRF, JDR, and AJD wrote and edited the manuscript. All authors have read and approved the manuscript.

\section{Funding}

This work was funded by the generous support of the Holden family and the support of the many patients and their families who contributed to the University of lowa Melanoma Research Fund. The funding sources were not involved in the design, data collection, data interpretation, or writing of this manuscript.

Availability of data and materials

All tools, including accompanying documentation, described in this manuscript can be acquired through GitHub (https://github.com/addupuy/IAS.git).

Ethics approval and consent to participate

Not applicable.

Consent for publication

Not applicable.

\section{Competing interests}

The authors declare that they have no competing interests.

\section{Author details}

${ }^{1}$ Department of Anatomy \& Cell Biology, Carver College of Medicine, University of lowa, lowa City, IA 52246, USA. ${ }^{2}$ Holden Comprehensive Cancer Center, University of lowa, lowa City, IA 52246, USA. ${ }^{3}$ Department of Anatomy \& Cell Biology, Cancer Biology Graduate Program, University of lowa, MERF, 375 Newton Road, lowa City, IA 3202, USA. 
Received: 17 April 2019 Accepted: 6 June 2019

Published online: 17 June 2019

\section{References}

1. Ammar I, Gogol-Doring A, Miskey C, Chen W, Cathomen T, Izsvak Z, Ivics Z. Retargeting transposon insertions by the adeno-associated virus rep protein. Nucleic Acids Res. 2012;40:6693-712.

2. Bard-Chapeau EA, Nguyen AT, Rust AG, Sayadi A, Lee P, Chua BQ, New LS, de Jong J, Ward JM, Chin CK, et al. Transposon mutagenesis identifies genes driving hepatocellular carcinoma in a chronic hepatitis B mouse model. Nat Genet. 2014:46:24-32.

3. Berquam-Vrieze KE, Nannapaneni K, Brett BT, Holmfeldt L, Ma J, Zagorodna O, Jenkins NA, Copeland NG, Meyerholz DK, Knudson CM, et al. Cell of origin strongly influences genetic selection in a mouse model of T-ALL. Blood. 2011;118:4646-56.

4. Bester AC, Lee JD, Chavez A, Lee YR, Nachmani D, Vora S, Victor J, Sauvageau M, Monteleone E, Rinn JL, et al. An integrated genome-wide CRISPRa approach to functionalize IncRNAs in drug resistance. Cell. 2018; 173:649-664 e620.

5. Brett BT, Berquam-Vrieze KE, Nannapaneni K, Huang J, Scheetz TE, Dupuy AJ. Novel molecular and computational methods improve the accuracy of insertion site analysis in sleeping beauty-induced tumors. PLoS One. 2011;6:e24668.

6. Chen HJ, Wei Z, Sun J, Bhattacharya A, Savage DJ, Serda R, Mackeyev Y, Curley SA, Bu P, Wang L, et al. A recellularized human colon model identifies cancer driver genes. Nat Biotechnol. 2016;34:845-51.

7. Collier LS, Carlson CM, Ravimohan S, Dupuy AJ, Largaespada DA. Cancer gene discovery in solid tumours using transposon-based somatic mutagenesis in the mouse. Nature. 2005;436:272-6.

8. Dupuy AJ. Transposon-based screens for cancer gene discovery in mouse models. Semin Cancer Biol. 2010;20:261-8.

9. Dupuy AJ, Akagi K, Largaespada DA, Copeland NG, Jenkins NA. Mammalian mutagenesis using a highly mobile somatic sleeping beauty transposon system. Nature. 2005;436:221-6.

10. Dupuy AJ, Rogers LM, Kim J, Nannapaneni K, Starr TK, Liu P, Largaespada DA, Scheetz TE, Jenkins NA, Copeland NG. A modified sleeping beauty transposon system that can be used to model a wide variety of human cancers in mice. Cancer Res. 2009;69:8150-6.

11. Feddersen CR, Schillo JL, Varzavand A, Vaughn HR, Wadsworth LS, Voigt AP, Zhu EY, Jennings BM, Mullen SA, Bobera J et al. 2019. Src-dependent DBL family members drive resistance to vemurafenib in human melanoma. bioRxiv doi:https://doi.org/10.1101/561597

12. Forsburg SL. The art and design of genetic screens: yeast. Nat Rev Genet. 2001:2:659-68.

13. Geurts AM, Yang Y, Clark KJ, Liu G, Cui Z, Dupuy AJ, Bell JB, Largaespada DA, Hackett PB. Gene transfer into genomes of human cells by the sleeping beauty transposon system. Mol Ther. 2003;8:108-17.

14. Gragerov A, Horie K, Pavlova M, Madisen L, Zeng H, Gragerova G, Rhode A, Dolka I, Roth P, Ebbert A, et al. Large-scale, saturating insertional mutagenesis of the mouse genome. Proc Natl Acad Sci U S A. 2007;104:14406-11.

15. Guo Y, Updegraff BL, Park S, Durakoglugil D, Cruz VH, Maddux S, Hwang TH, O'Donnell KA. Comprehensive ex vivo transposon mutagenesis identifies genes that promote growth factor Independence and Leukemogenesis. Cancer Res. 2016;76:773-86.

16. Hart T, Chandrashekhar M, Aregger M, Steinhart Z, Brown KR, MacLeod G, Mis M, Zimmermann M, Fradet-Turcotte A, Sun S, et al. High-resolution CRISPR screens reveal fitness genes and genotype-specific Cancer liabilities. Cell. 2015;163:1515-26

17. Jorgensen EM, Mango SE. The art and design of genetic screens: caenorhabditis elegans. Nat Rev Genet. 2002;3:356-69.

18. Joung J, Konermann S, Gootenberg JS, Abudayyeh OO, Platt RJ, Brigham MD, Sanjana NE, Zhang F. Genome-scale CRISPR-Cas9 knockout and transcriptional activation screening. Nat Protoc. 2017;12:828-63.

19. Kandel ES, Lu T, Wan Y, Agarwal MK, Jackson MW, Stark GR. Mutagenesis by reversible promoter insertion to study the activation of NF-kappaB. Proc Natl Acad Sci U S A. 2005;102:6425-30.

20. Keng WW, Sia D, Sarver AL, Tschida BR, Fan D, Alsinet C, Sole M, Lee WL, Kuka TP, Moriarity BS, et al. Sex bias occurrence of hepatocellular carcinoma in Poly7 molecular subclass is associated with EGFR. Hepatology. 2013;57:120-30.

21. Kim D, Langmead B, Salzberg SL. HISAT: a fast spliced aligner with low memory requirements. Nat Methods. 2015;12:357-60.
22. Kizilors A, Pickard MR, Schulte CE, Yacqub-Usman K, McCarthy NJ, Gan SU, Darling D, Gaken J, Williams GT, Farzaneh F. Retroviral insertional mutagenesis implicates E3 ubiquitin ligase RNF168 in the control of cell proliferation and survival. Biosci Rep. 2017. https://doi.org/10.1042/BSR20170843.

23. Kodama T, Newberg JY, Kodama M, Rangel R, Yoshihara K, Tien JC, Parsons PH, Wu H, Finegold MJ, Copeland NG, et al. Transposon mutagenesis identifies genes and cellular processes driving epithelial-mesenchymal transition in hepatocellular carcinoma. Proc Natl Acad Sci U S A. 2016;113:E3384-93.

24. Koike-Yusa H, Li Y, Tan EP, Velasco-Herrera Mdel C, Yusa K. Genome-wide recessive genetic screening in mammalian cells with a lentiviral CRISPRguide RNA library. Nat Biotechnol. 2014;32:267-73.

25. Konermann S, Brigham MD, Trevino AE, Joung J, Abudayyeh OO, Barcena C, Hsu PD, Habib N, Gootenberg JS, Nishimasu H, et al. Genome-scale transcriptional activation by an engineered CRISPR-Cas9 complex. Nature. 2015;517:583-8.

26. Le Sage C, Lawo S, Panicker P, Scales TME, Rahman SA, Little AS, McCarthy NJ, Moore JD, Cross BCS. Dual direction CRISPR transcriptional regulation screening uncovers gene networks driving drug resistance. Sci Rep. 2017;7:17693.

27. Liang Q, Kong J, Stalker J, Bradley A. Chromosomal mobilization and reintegration of sleeping beauty and PiggyBac transposons. Genesis. 2009;47:404-8.

28. Ling A, Gruener RF, Fessler J, Huang RS. More than fishing for a cure: the promises and pitfalls of high throughput cancer cell line screens. Pharmacol Ther. 2018;191:178-89.

29. Mann KM, Ward JM, Yew CC, Kovochich A, Dawson DW, Black MA, Brett BT, Sheetz TE, Dupuy AJ, Australian Pancreatic Cancer Genome I, et al. Sleeping beauty mutagenesis reveals cooperating mutations and pathways in pancreatic adenocarcinoma. Proc Natl Acad Sci U S A. 2012;109:5934-41.

30. Mann MB, Black MA, Jones DJ, Ward JM, Yew CC, Newberg JY, Dupuy AJ, Rust AG, Bosenberg MW, McMahon M, et al. Transposon mutagenesis identifies genetic drivers of Braf(V600E) melanoma. Nat Genet. 2015:47:486-95.

31. Martin M. Cutadapt removes adapter sequences from high-throughput sequencing reads. EMBnetjournal. 2011;17:10.

32. Mates L, Chuah MK, Belay E, Jerchow B, Manoj N, Acosta-Sanchez A, Grzela DP, Schmitt A, Becker K, Matrai J, et al. Molecular evolution of a novel hyperactive sleeping beauty transposase enables robust stable gene transfer in vertebrates. Nat Genet. 2009:41:753-61.

33. Montero-Conde C, Leandro-Garcia LJ, Chen X, Oler G, Ruiz-Llorente S, Ryder M, Landa I, Sanchez-Vega F, La K, Ghossein RA, et al. Transposon mutagenesis identifies chromatin modifiers cooperating with Ras in thyroid tumorigenesis and detects ATXN7 as a cancer gene. Proc Natl Acad Sci U S A. 2017;114:E4951-60.

34. Morris SM, Davison J, Carter KT, O'Leary RM, Trobridge P, Knoblaugh SE, Myeroff LL, Markowitz SD, Brett BT, Scheetz TE, et al. Transposon mutagenesis identifies candidate genes that cooperate with loss of transforming growth factor-beta signaling in mouse intestinal neoplasms. Int J Cancer. 2017;140:853-63.

35. O'Donnell KA. Advances in functional genetic screening with transposons and CRISPR/Cas9 to illuminate cancer biology. Curr Opin Genet Dev. 2018;49:85-94.

36. Poulikakos PI, Persaud $Y$, Janakiraman M, Kong X, Ng C, Moriceau G, Shi H, Atefi M, Titz B, Gabay MT, et al. RAF inhibitor resistance is mediated by dimerization of aberrantly spliced BRAF(V600E). Nature. 2011;480:387-90.

37. Quintana RM, Dupuy AJ, Bravo A, Casanova ML, Alameda JP, Page A, SanchezViera M, Ramirez A, Navarro M. A transposon-based analysis of gene mutations related to skin cancer development. J Invest Dermatol. 2013;133:239-48.

38. Rauscher B, Heigwer F, Breinig M, Winter J, Boutros M. GenomeCRISPR - a database for high-throughput CRISPR/Cas9 screens. Nucleic Acids Res. 2017;45:D679-86.

39. Riordan JD, Drury LJ, Smith RP, Brett BT, Rogers LM, Scheetz TE, Dupuy AJ. Sequencing methods and datasets to improve functional interpretation of sleeping beauty mutagenesis screens. BMC Genomics. 2014;15:1150.

40. Riordan JD, Feddersen CR, Tschida BR, Beckmann PJ, Keng W, Linden MA, Amin K, Stipp CS, Largaespada DA, Dupuy AJ. Chronic liver injury alters driver mutation profiles in hepatocellular carcinoma in mice. Hepatology. 2018;67:924-39.

41. Rogers LM, Olivier AK, Meyerholz DK, Dupuy AJ. Adaptive immunity does not strongly suppress spontaneous tumors in a sleeping beauty model of cancer. J Immunol. 2013;190:4393-9.

42. Schmidt EE, Pelz O, Buhlmann S, Kerr G, Horn T, Boutros M. GenomeRNAi: a database for cell-based and in vivo RNAi phenotypes, 2013 update. Nucleic Acids Res. 2013:41:D1021-6.

43. Shalem O, Sanjana NE, Hartenian E, Shi X, Scott DA, Mikkelson T, Heckl D, Ebert BL, Root DE, Doench JG, et al. Genome-scale CRISPR-Cas9 knockout screening in human cells. Science (New York, NY). 2014;343:84-7. 
44. St Johnston D. The art and design of genetic screens: Drosophila melanogaster. Nat Rev Genet. 2002;3:176-88.

45. Suarez-Cabrera C, Quintana RM, Bravo A, Casanova ML, Page A, Alameda JP, Paramio JM, Maroto A, Salamanca J, Dupuy AJ, et al. A transposon-based analysis reveals RASA1 is involved in triple-negative breast Cancer. Cancer Res. 2017;77:1357-68.

46. Takeda H, Wei Z, Koso H, Rust AG, Yew CC, Mann MB, Ward JM, Adams DJ, Copeland NG, Jenkins NA. Transposon mutagenesis identifies genes and evolutionary forces driving gastrointestinal tract tumor progression. Nat Genet. 2015;47:142-50.

47. Tschida BR, Temiz NA, Kuka TP, Lee LA, Riordan JD, Tierrablanca CA, Hullsiek R, Wagner S, Hudson WA, Linden MA, et al. Sleeping beauty insertional mutagenesis in mice identifies drivers of steatosis-associated hepatic tumors. Cancer Res. 2017;77:6576-88.

48. Vigdal TJ, Kaufman CD, Izsvak Z, Voytas DF, Ivics Z. Common physical properties of DNA affecting target site selection of sleeping beauty and other Tc1/mariner transposable elements. J Mol Biol. 2002;323:441-52.

49. Wang T, Wei JJ, Sabatini DM, Lander ES. Genetic screens in human cells using the CRISPR-Cas9 system. Science (New York, NY). 2014;343:80-4.

50. Whittaker SR, Theurillat JP, Van Allen E, Wagle N, Hsiao J, Cowley GS, Schadendorf D, Root DE, Garraway LA. A genome-scale RNA interference screen implicates NF1 loss in resistance to RAF inhibition. Cancer discovery. 2013;3:350-62.

51. Woodard LE, Li X, Malani N, Kaja A, Hice RH, Atkinson PW, Bushman FD, Craig NL, Wilson MH. Comparative analysis of the recently discovered hAT transposon TcBuster in human cells. PLoS One. 2012;7:e42666.

52. Wu X, Northcott PA, Dubuc A, Dupuy AJ, Shih DJ, Witt H, Croul S, Bouffet E, Fults DW, Eberhart CG, et al. Clonal selection drives genetic divergence of metastatic medulloblastoma. Nature. 2012;482:529-33.

53. Yant SR, Wu X, Huang Y, Garrison B, Burgess SM, Kay MA. High-resolution genome-wide mapping of transposon integration in mammals. Mol Cell Biol. 2005;25:2085-94.

54. Yusa K, Zhou L, Li MA, Bradley A, Craig NL. A hyperactive piggyBac transposase for mammalian applications. Proc Natl Acad Sci U S A. 2011;108:1531-6.

55. Zanesi N, Balatti V, Riordan J, Burch A, Rizzotto L, Palamarchuk A, Cascione L, Lagana A, Dupuy AJ, Croce CM, et al. A sleeping beauty screen reveals NFkB activation in CLL mouse model. Blood. 2013;121:4355-8.

\section{Publisher's Note}

Springer Nature remains neutral with regard to jurisdictional claims in published maps and institutional affiliations.

Ready to submit your research? Choose BMC and benefit from:

- fast, convenient online submission

- thorough peer review by experienced researchers in your field

- rapid publication on acceptance

- support for research data, including large and complex data types

- gold Open Access which fosters wider collaboration and increased citations

- maximum visibility for your research: over $100 \mathrm{M}$ website views per year

At BMC, research is always in progress.

Learn more biomedcentral.com/submissions 